\title{
Dynamic Optimal Law Enforcement with Learning
}

\author{
Mohamed JELLAL * \\ CES Université de Rabat \\ Nuno GAROUPA ${ }^{\dagger}$ \\ Universitat Pompeu Fabra, Barcelona
}

June 1999

${ }^{*}$ CES, Department of Economics, University of Mohammed V, Rabat, Morocco. Email: Jellal@compuserve.com

†Department d'Economia i Empresa, Universitat Pompeu Fabra, Ramon Trias Fargas 25-27, 08005 Barcelona, Spain. Phone: 34-93-542 2639. Fax: 34-93-542 1746. Email: nuno.garoupa@econ.upf.es 


\begin{abstract}
We incorporate the process of enforcement learning by assuming that the agency's current marginal cost is a decreasing function of its past experience of detecting and convicting. The agency accumulates data and information (on criminals, on opportunities of crime) enhancing the ability to apprehend in the future at a lower marginal cost.

We focus on the impact of enforcement learning on optimal stationary compliance rules. In particular, we show that the optimal stationary fine could be less-than-maximal and the optimal stationary probability of detection could be higher-than-otherwise.
\end{abstract}

JEL: K4.

Keywords: fine, probability of detection and punishment, learning. 


\section{Introduction}

Economic theory of law enforcement has primarily been built on static models. Since Becker (1968) seminal paper, the economic theory of compliance and deterrence has been confined to static analysis even though the importance of dynamics as been recognized long time ago. The fundamental problem of a static model is the exclusion of learning and recidivistic behavior, as individuals are allowed to break the law only once.

Once we attend at a dynamic model rather than a static model of compliance, we must consider the source of dynamics. In other words, a fundamental issue is to understand why decisions by both potential offenders and the government at a given period are history path dependent. Different sources of history path dependence have been considered in the literature.

One source of dynamics is that the pool of potential offenders at time $t$ depends on the pool of offenders at time $t-1$ because (a) some offenders are detected and punished at time $t-1$ and can no longer commit an offense at time $t$ (e.g. they are imprisoned), (b) the offense will continue until detected, (c) offenders solve an optimal stopping problem by choosing a path of offense rate over a temporal horizon, or (d) gains from illegal activities are path dependent (e.g. criminal know-how). ${ }^{1}$ Compliance rules at time $t-1$ affect the pool of potential offenders at time $t$. The government should choose compliance rules at time $t$ that optimally deter offenses at the current period and at future periods.

A second source of dynamics comes from the fact that potential offender's perceptions are determined endogenously incorporating information available to them. This information is generated within the economy as time goes by. ${ }^{2}$ Compliance rules should be condition on the learning dynamics. As an example, raising the probability of detection increases the number of occasions in which offenders get caught giving them more information about law enforcement.

Essentially the literature has considered 'supply side' dynamics, that

\footnotetext{
${ }^{1}$ Davis (1988), Leung (1991, 1995), Nash (1991), Polinsky and Shavell (1998).

${ }^{2}$ Sah (1991), Ben-Shahar (1997).
} 
is, the path dependence is directly related to potential offenders. In this paper, we address 'demand side' dynamics, that is, the path dependence is related to the enforcement agency and not to the offenders. We incorporate the process of enforcement learning by assuming that the agency's current marginal cost is a decreasing function of its past experience of detecting and convicting. The agency accumulates data and information (on criminals, on opportunities of crime) enhancing the ability to apprehend in the future at a lower marginal cost.

We focus on the impact of enforcement learning on optimal stationary compliance rules. In particular, we show that the optimal stationary fine could be less-than-maximal and the optimal stationary probability of detection could be higher-than-otherwise. If the present value of reducing the cost of enforcement tomorrow because of learning more than compensates the present cost of enforcement at the steady-state, then the government should set a higher-than-otherwise probability and reduce the fine appropriately.

\section{A Model of Law Enforcement}

Consider an economy of risk-neutral individuals who choose whether to commit an act that benefits the actor by $b$ and harms the rest of society by $h$. The policy maker does not know individual's gain $b$, but knows the distribution of parties by type described by a distribution $G(b)$ with support $[0, B]$, with a positive density $g(b)$. We suppose that $h<B$, that is, not every offense is socially undesirable.

The government at time $t$ chooses a sanction $f(t)$ and a probability of detection and conviction $p(t)$. The expenditure on detection and conviction to achieve a probability $p(t)$ is given by $C(E(t)) p(t)$, where $C($.$) is the marginal$ cost and $E(t)$ is a measure of past enforcement experience. In particular, we assume:

$$
E(t)=\rho \int_{-\infty}^{t} p(\tau) e^{-\rho(t-\tau)} d \tau
$$

Hence, the measure of past experience is developed over past probabilities $p(\tau), \tau \leq t$. Our measure $E(t)$ is a weighted sum of past probabilities $p(\tau)$ 
(for $\tau \leq t$ ) with exponentially declining weights given to more distant values of $p(\tau)$, where $\rho>0$ is the rate of memory. More distant history deterrence events carry less weight in the learning mechanism.

Following Ryder and Heal (1973), the evolution of $E(t)$ is given by:

$$
\dot{E}(t)=\rho(p(t)-E(t))
$$

From (2), we can observe that if the current probability of detection and apprehension $p(t)$ is more than the weighted sum of past probabilities $E(t)$, we have $\dot{E}(t)>0$. Conversely, reducing the probability of detection and apprehensions affects negatively deterrence experience. We could think that with less detection and apprehension, the agency forgets about past experience.

Regarding the cost function we assume that $C(0)=C>0, C^{\prime}(E(t))<$ 0 , and $C^{\prime \prime}(E(t)) \geq 0$. These assumptions pose that the agency must detect and convict offenders in order to learn and the cost declines with the habit of detecting but at a decreasing rate. While the agency's technology (cost) at any point in time displays constant returns to scale, it is characterized by dynamic economies to scale.

At time $t$, a risk-neutral individual commits an offense if and only if $b \geq p(t) f(t)$. We assume that both the individual's gain $b$ and the magnitude of harm $h$ are time invariant.

In the optimal law enforcement literature, social welfare at time $t$ generally equals the sum of individuals' expected utilities minus the harm caused by offenses minus expenditure on law enforcement ${ }^{3}$ :

$$
W(t)=\int_{p(t) f(t)}^{B}(b-h) d G(b)-C(E(t)) p(t)
$$

The monetary sanction is assumed to be costless to impose as conventional in the law enforcement literature, and $F$ is the maximal feasible sanction.

\footnotetext{
${ }^{3}$ See Garoupa (1997) and Polinsky and Shavell (1999). It is conventional in this literature to include all gains in social welfare. Some argue that the offender's gains should be excluded for moral reasons.
} 
The policy maker maximizes the following objective function:

$$
\int_{0}^{\infty}\left\{\int_{p(t) f(t)}^{B}(b-h) d G(b)-C(E(t)) p(t)\right\} e^{-r t} d t
$$

where $r>0$ is the discount rate. The maximization problem is subject to the evolution of $E(t)$ described by (2), the maximal feasible sanction constraint $f(t) \leq F$, and $E(0)=E_{0} \geq 0$. Here we have typically an optimal control problem where the control variables are $p(t)$ and $f(t)$, and the state variable is $E(t)$. If we denote by $\lambda(t)$ the costate variable associated with $E(t)$, the Hamiltonian function $\mathcal{H}$ can be expressed as:

$\mathcal{H}=\int_{p(t) f(t)}^{B}(b-h) d G(b)-C(E(t)) p(t)+\lambda(t) \rho(p(t)-E(t))+\mu(t)(F-f(t))$

where $\mu$ is the usual Lagrangean multiplier.

Let us define the following elasticity:

$$
\epsilon=-\frac{C^{\prime}(E)}{C(E)} E
$$

which is the elasticity of learning with respect to detection and conviction.

Proposition 1 (a) If $\epsilon\left(p^{*}\right) \geq 1+r / \rho$ and $F>h$, the optimal steady-state fine is less-than-maximal. (b) If $\epsilon\left(p^{*}\right)<1+r / \rho$ or $F \leq h$, the optimal steady-state fine is maximal.

\section{Proof}

Following Chiang (1992) and according to the maximum principle, the control variables $p(t)$ and $f(t)$ must be chosen so as to maximize $\mathcal{H}$ at each time $t$, given the current values of the state and costate variables, $E(t)$ and $\lambda(t)$. If one assumes continuous differentiability of the Hamiltonian, we have:

$$
\frac{\partial \mathcal{H}}{\partial p(t)}=f(t)(h-p(t) f(t)) g(.)-C(E(t))+\lambda(t) \rho=0
$$




$$
\frac{\partial \mathcal{H}}{\partial f(t)}=p(t)(h-p(t) f(t)) g(.)-\mu(t)=0
$$

Furthermore, optimal control theory imposes two necessary conditions that the costate variable $\lambda$ must satisfy. The first of these conditions is:

$$
\begin{aligned}
\dot{\lambda}(t) & =r \lambda(t)-\frac{\partial \mathcal{H}}{\partial E(t)} \\
& =(r+\rho) \lambda(t)+C^{\prime}(E(t)) p(t)
\end{aligned}
$$

and the second of these constraints consists of the following transversality condition:

$$
\lim _{t \rightarrow+\infty} e^{-r t} \lambda(t) E(t)=0
$$

We also need to invoke the Kuhn-Tucker conditions to deal with the inequality constraint. We must have $f(t) \leq F, \mu(t) \geq 0$, and $\mu(t)(F-f(t))=$ 0 .

We are interested in the steady-state solution of this problem. The stationary state to this problem is described by $\dot{E}(t)=\dot{\lambda}(t)=0$. At the steady-state solution, we must have:

$$
\begin{gathered}
\dot{E}=\rho(p-E)=0 \\
\dot{\lambda}=(r+\rho) \lambda+C^{\prime}(E) p=0
\end{gathered}
$$

Thus, $E^{*}=p^{*}$ and $\lambda^{*}=-C^{\prime}\left(p^{*}\right) p^{*} /(r+\rho)=C\left(p^{*}\right) \epsilon /(r+\rho)$.

Using these two results, we can re-write (5) and (6) as:

$$
\begin{gathered}
f^{*}\left(h-p^{*} f^{*}\right) g(.)=C\left(p^{*}\right)\left(1-\frac{\rho}{r+\rho} \epsilon\right) \\
p^{*}\left(h-p^{*} f^{*}\right) g(.)=\mu^{*}
\end{gathered}
$$

If a less-than-maximal sanction is the optimal solution, then it must be the case that $\mu^{*}=0$, the constraint is not binding. From (6), we have that 
$p^{*} f^{*}=h$. Therefore, when $F \leq h$, the optimal fine cannot be less-thanmaximal. A less-than-maximal sanction is possible if and only if $F>h$.

Using these results in (5), we conclude that it if $\epsilon\left(p^{*}\right) \geq 1+r / \rho$, we have a less-tha-maximal fine. The result follows from combining both $\epsilon\left(p^{*}\right) \geq 1+r / \rho$ and $F>h$.

The marginal social cost of imposing a monetary sanction is zero and the marginal social cost of setting a probability of detection and punishment at the steady-state is given by $C().\left(1-\frac{\rho}{r+\rho} \epsilon\right)$. As long as $\epsilon\left(p^{*}\right)<1+r / \rho$, the marginal cost of setting a probability of detection and punishment at the steady-state is positive. In this case, the usual high-fine-low-probability argument applies: we should take the sanction to its maximum and complement its deterrent effect with a lower-than-otherwise probability. From Polinsky and Shavell (1999), we also know that the expected fine is less than the magnitude of harm $h$. In other words, there will be offenses which socially yield a negative surplus and nevertheless will be committed because it is too costly to deter them.

Take the case such that $\epsilon=1+r / \rho$. The marginal social cost of setting a probability of detection and punishment at the steady-state is zero. Therefore, the government is indifferent between probability and fine. Furthermore, unlike Polinsky and Shavell (1999), the expected sanction is equal to the magnitude of harm $h$. At steady-state, we have efficient deterrence. Only offenses that yield a socially positive surplus are committed.

The last case to consider is $\epsilon>1+r / \rho$. The marginal cost of setting a probability of detection and punishment at the steady-state is negative. Therefore, the policy maker will take the probability to one and complement with the fine such that $f^{*}=h$.

The marginal social cost of setting a probability of detection and apprehension at steady-state assumes a critical role on the result. This marginal cost has two terms. The first term, $C($.$) , is the usual in the law enforcement$ literature and the driving force of the high-fine-low-probability result. The second term, $\rho \lambda^{*}$, measures the present value of reducing the marginal social cost of the probability of detection and punishment tomorrow. If the present value of reducing the marginal social cost of the probability of detection and 
punishment tomorrow more than compensates the present marginal social cost of that probability at the steady-state, then the government should set a probability equal to one.

Proposition 2 The condition for a less-than-maximal fine at the steadystate is more likely to be satisfied if (a) the elasticity of learning with respect to detection and conviction is high, (b) the discount rate $r$ is small, and (c) the rate of memory $\rho$ is high.

\section{Proof}

The result follows trivially from $\epsilon\left(p^{*}\right) \geq 1+r / \rho$.

A less-than-maximal fine at the steady-state is a consequence of enforcement learning being very valuable for the government to the point of setting a higher-than-otherwise probability. Proposition 2 states the conditions under which setting a higher-than-otherwise probability is valuable for the government: (a) when the probability affects decisively the learning process, (b) when the government cares for the future, and (c) when there is learning. In particular, when $\rho=0$ (no memory of past experience) or when $\epsilon=0$ (independent history), we have the standard result $f^{*}\left(h-p^{*} f^{*}\right) g()=.C\left(p^{*}\right)$.

Proposition 3 The optimal steady-state probability decreases with $r$ and increases with $\rho$.

\section{Proof}

The result follows from the observation that the marginal cost of the probability increases with $r$ and decreases with $\rho$.

A higher-than-otherwise probability is set by the government as the discount rate decreases (as the future becomes more important) and the rate

of memory increases (as the gains from learning become more important at the moment of setting the current probability). 


\section{Conclusion}

We have shown that a less-than-maximal sanction at steady-state is possible as a response to a dynamic feature of enforcement policy. The marginal cost of enforcement depends on a measure of learning provided by past enforcement experience. Setting a higher probability of detection and apprehension at time $t-1$ provides a further gain in reducing the marginal cost of enforcement at time $t$. We have shown that this effect leads to a higherthan-otherwise probability at steady-state. Aiming at optimal deterrence, the government could have to reduce the sanction to a less-than-maximal fine.

In our model, the possibility of a less-than-maximal fine at steady-state is somewhat extreme since it only happens if the gains from learning more than compensate the current marginal cost. In a model like the one by Kaplow (1990), where nonmonetary sanctions are considered, there is a positive social marginal cost from imposing a sanction. As a consequence, our result should be re-interpreted as suggesting a less-than-otherwise sanction at steady-state because of a dynamic feature of enforcement policy.

An interesting extension of the model is to consider that an imprisonment term could also benefit the government in providing information about criminal opportunities. Setting longer imprisonment terms at time $t-1$ reduces the cost of enforcement at time $t$. Consequently, the optimal imprisonment term at steady-state could be higher-than-otherwise. Polinsky and Shavell (1984) have argued that the monetary sanction should be taken to its highest value, and an imprisonment term should be used to complement it when the maximal fine is not very large. Using the rationale we have provided in this paper, we could have a higher-than-otherwise imprisonment term with a less-than-otherwise fine at steady-state if the future marginal gains from learning more than compensate the current marginal cost of imprisonment.

\section{References}

1. G. S. Becker, 1968, Crime and Punishment: An Economic Approach, 
Journal of Political Economy, Vol. 76. Pages 169-217.

2. O. Ben-Shahar, 1997, Playing Without a Rulebook: Optimal Enforcement When Individuals Learn the Penalty Only by Committing the Crime, International Review of Law and Economics, Vol. 17. Pages $410-421$.

3. A. C. Chiang, 1992, Elements of Dynamic Optimization, MacGraw-Hill International Editions: New York.

4. M. Davis, 1988, Time and Punishment: An Intertemporal Model of Crime, Journal of Political Economy, Vol. 96. Pages 383-390.

5. N. Garoupa, 1997, The Theory of Optimal Law Enforcement, Journal of Economic Surveys, Vol. 11. Pages 267-295.

6. L. Kaplow, 1990, A Note on the Optimal Use of Nonmonetary Sanctions, Journal of Law and Economics, Vol. 42. Pages 245-247.

7. S. F. Leung, 1991, How to Make the Fine Fit the Corporate Crime?, Journal of Public Economics, Vol. 45. Pages 243-256.

8. S. F. Leung, 1995, Dynamic Deterrence Theory, Economica, Vol. 62. Pages 65-88.

9. J. Nash, 1991, To Make the Punishment Fit the Crime: The Theory and Statistical Estimation of a Multi-Period Optimal Deterrence Model, International Review of Law and Economics, Vol. 11. Pages 101-110.

10. A. M. Polinsky and S. Shavell, 1984, The Optimal Use of Fines and Imprisonment, Journal of Public Economics, Vol. 24. Pages 89-99.

11. A. M. Polinsky and S. Shavell, 1998, On Offense History and the Theory of Deterrence, International Review of Law and Economics, Vol. 18. Pages 305-324.

12. A. M. Polinsky and S. Shavell, 1999, The Economic Theory of Public Enforcement of Law, Journal of Economic Literature, forthcoming. 
13. H. E. Ryder and G. M. Heal, 1973, Optimal Growth with Intertemporal Dependent Preferences, Review of Economic Studies, Vol. 40. Pages 1-31.

14. R. K. Sah, 1991, Social Osmosis and Patterns of Crime, Journal of Political Economy, Vol. 99. Pages 1272-1295.

15. S. Shavell, 1993, The Optimal Structure of Law Enforcement, Journal of Law and Economics, Vol. 36. Pages 255-287. 